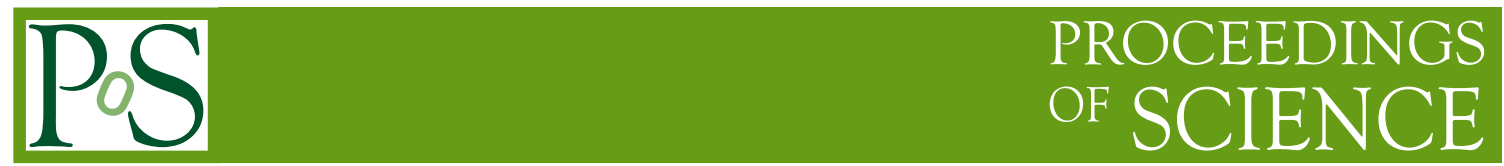

\title{
Vector boson production in association with jets from CMS
}

\author{
Thomas Mastrianni Perry* \\ University of Wisconsin - Madison \\ E-mail: t.perryecern.ch
}

The production of vector bosons $(V=W, Z$ or $\gamma$ ) in association with jets is a stringent test of perturbative QCD and is a background process in searches for new physics. Total and differential cross-section measurements of vector bosons produced in association with jets and heavy flavor quarks in proton-proton collisions at the LHC are presented. The measurements are compared to next-to leading order calculations and event simulations that devise matrix element calculations interfaced with parton showers.

XXIII International Workshop on Deep-Inelastic Scattering,

27 April - May 12015

Dallas, Texas

* Speaker.

${ }^{\dagger}$ The Standard Model Physics Group at CMS 


\section{Introduction}

The production of vector bosons $\left(V=W, Z\right.$ or $\gamma$ where $\left.Z=Z / \gamma^{*}\right)$ associated with jets is predicted by the standard model and serves as a mechanism for probing our understanding of quantum chromodynamics (QCD) in the perturbative regime by providing input for theoretical calculations as they reach new limits in precision. Through the comparison between measurement and the monte carlo (MC) simulations made using various generators and parton distribution functions (PDFs), important feedback is also given with regard to the validity of different MC techniques. The measurements presented in these proceedings were made by the Compact Muon Solenoid (CMS) experiment at the Large Hadron Collider (LHC) using samples of proton-proton collisions at 7 and $8 \mathrm{TeV}$. An overview of measurements and comparisons with MC predictions is shown in Figure 1, where cross section measurements can be seen to span five orders of magnitude and include heavy and light flavor jets. The scope of these proceedings will cover only the most recent measurements, including $V+b(\bar{b}), Z+$ jets, $\gamma+$ jets, and combined $Z / \gamma+$ jets ratio results.

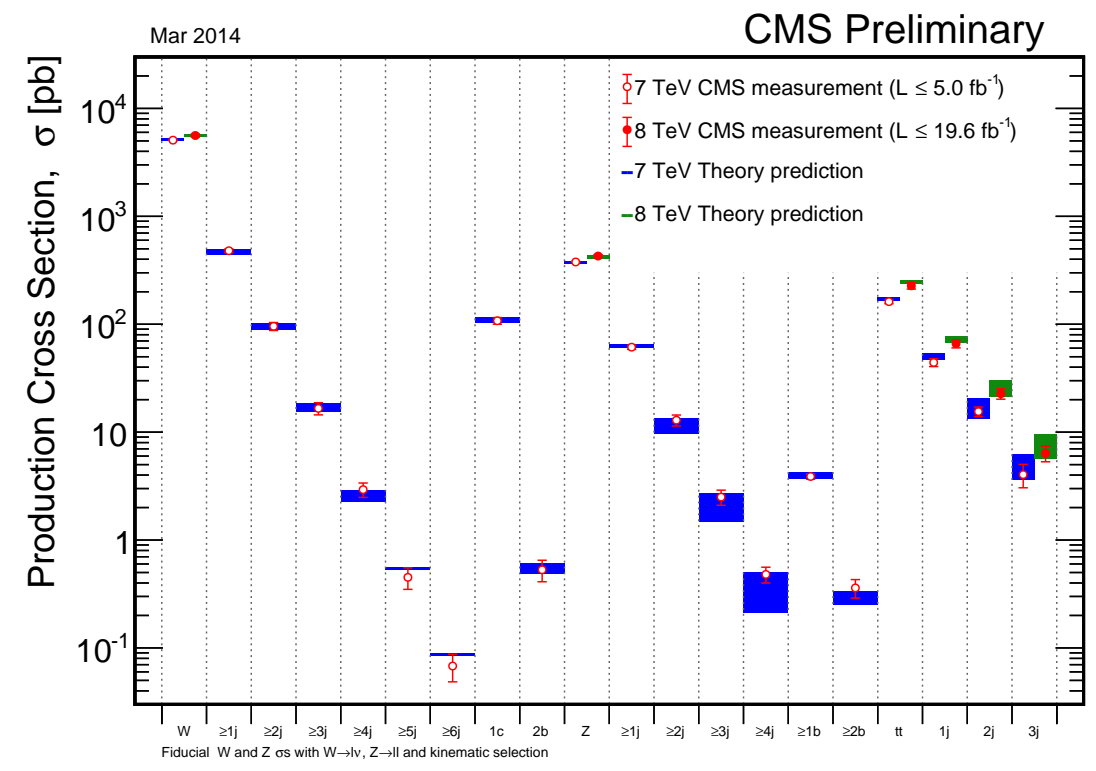

Figure 1: A summary of the CMS measurements of vector boson production in association with jets.

\section{Vector boson production in association with heavy flavor jets}

The production of $\mathrm{b}$ quark pairs along with $\mathrm{W}$ or $\mathrm{Z}$ bosons is an area of active research and has a historical precident of being difficult to model. With almost $95 \%$ of $b \bar{b}$ pairs being produced via gluon splitting, measurements of $V+b \bar{b}$ cross sections provide input to our understanding of the $\mathrm{b}$ quark content of the proton. Additionally, associated electroweak vector boson production with the Higgs boson, where the Higgs decays to a $b \bar{b}$ pair has $V+b \bar{b}$ as an irreducible background, and many searches beyond the standard model also explore phase spaces involving isolated lepton(s), missing transverse energy (MET) and heavy flavor quark(s), making $V+b \bar{b}$ an interesting process to probe experimentally. 


\begin{tabular}{l|c|ccccc}
\hline Cross section & Measured & $\begin{array}{c}\text { MADGRAPH } \\
(5 \mathrm{~F})\end{array}$ & $\begin{array}{c}\text { aMC@NLO } \\
(5 \mathrm{~F})\end{array}$ & $\begin{array}{c}\text { MCFM } \\
\text { (parton level) }\end{array}$ & $\begin{array}{c}\text { MADGRAPH } \\
(4 \mathrm{~F})\end{array}$ & $\begin{array}{c}\text { aMC@NLO } \\
(4 \mathrm{~F})\end{array}$ \\
\hline$\sigma_{\mathrm{Z}+1 \mathrm{~b}}(\mathrm{pb})$ & $3.52 \pm 0.02 \pm 0.20$ & $3.66 \pm 0.22$ & $3.70_{-0.26}^{+0.23}$ & $3.03_{-0.36}^{+0.30}$ & $3.11_{-0.81}^{+0.47}$ & $2.36_{-0.37}^{+0.47}$ \\
$\sigma_{\mathrm{Z}+2 \mathrm{~b}}(\mathrm{pb})$ & $0.36 \pm 0.01 \pm 0.07$ & $0.37 \pm 0.07$ & $0.29_{-0.04}^{+0.04}$ & $0.29_{-0.04}^{+0.04}$ & $0.38_{-0.10}^{+0.06}$ & $0.35_{-0.06}^{+0.08}$ \\
$\sigma_{\mathrm{Z}+\mathrm{b}}(\mathrm{pb})$ & $3.88 \pm 0.02 \pm 0.22$ & $4.03 \pm 0.24$ & $3.99_{-0.29}^{+0.25}$ & $3.23_{-0.40}^{+0.34}$ & $3.49_{-0.91}^{+0.52}$ & $2.71_{-0.41}^{+0.52}$ \\
\hline$\sigma_{\mathrm{Z}+\mathrm{b} / \mathrm{Z}+\mathrm{j}}(\%)$ & $5.15 \pm 0.03 \pm 0.25$ & $5.35 \pm 0.11$ & $5.38_{-0.39}^{+0.34}$ & $4.75_{-0.27}^{+0.24}$ & $4.63_{-1.21}^{+0.69}$ & $3.65_{-0.55}^{+0.70}$ \\
\hline
\end{tabular}

Table 1: Results of the measurement of cross sections for the production of a $\mathrm{Z}$ boson with exactly one $\mathrm{b}$ jet, with at least two $\mathrm{b}$ jets, with at least one $\mathrm{b}$ jet, and the ratio with respect to at least one jet of any flavor. Statistical and systematic uncertainties are included on the measurement and MADGRAPH, MCFM and aMC@NLO include uncertainties due to scale variations.

Using data collected at $\sqrt{s}=7 \mathrm{TeV}$, comparisons were made against MadGraph 5 matrix element (ME) calculations interfaced with Pythia 6 Tune $\mathrm{Z} 2$ for parton showering (PS) and Geant 4 for CMS detector simulation. The MC predictions compared to in the $W+b \bar{b}$ channel used a five-flavor $(5 \mathrm{~F})$ scheme, in which the five lowest mass quarks, including the $\mathrm{b}$ quark are taken to be massless [1]. The measurements in the $Z+b \bar{b}$ phase space were compared to predictions using both the 5F and four-flavor (4F) schemes using MadGraph as well as aMC@NLO and MCFM at the parton level [2].

In the $Z$ channel, two tightly isolated leptons of the same flavor and opposite charge were required to each have transverse momentum $p_{T}^{l}>20 \mathrm{GeV}$ and fall within the pseudorapidity range $\left|\eta^{l}\right|<2$.4. Additionally, jets were required which had $p_{T}^{j}>25 \mathrm{GeV}$ and $\left|\eta^{j}\right|<2.1$, satisfying high efficiency Simple Secondary Vertex (SSV) b Tagging requirements. The sample was divided into disjoint categories of exactly 1 jet which has a $\mathrm{b}$ Tag $(Z+1 b)$ and at least $2 \mathrm{~b}$ Tagged jets $(Z+2 b)$. An inclusive $Z+b$ cross section measurement was also made and the results of these comparisons are listed in Table 1.

In the $W+b \bar{b}$ channel, exactly one isolated muon was required with $p_{T}^{\mu}>25 \mathrm{GeV}$ and $\left|\eta^{\mu}\right|<2.1$ along with exactly two jets, $p_{T}^{j}>25 \mathrm{GeV}$ and $\left|\eta^{j}\right|<2.4$, both of which b Tagged using the Combined Secondary Vertex (CSV) tight working point. To reconstruct the $W$ and reject multijet (QCD) events, missing energy was also required via a selection on the transverse mass of the muon/MET pair, $m_{T}>45 \mathrm{GeV}$. Ultimately the measured cross section and prediction by MCFM are

\begin{tabular}{c|c}
\multicolumn{2}{c}{$\sigma(p p \rightarrow W+b \bar{b}) \times B(W \rightarrow \mu v)$} \\
\hline measured & $0.53 \pm 0.05$ (stat) \pm 0.09 (syst) \pm 0.06 (theo.) \pm 0.01 (lumi) pb \\
predicted & $0.55 \pm 0.03$ (MCFM) \pm 0.01 (had.) \pm 0.05 (DPS) pb
\end{tabular}

In both the $W+b \bar{b}$ and $Z+b$ channels, the measured cross sections are in agreement with the predictions of the SM using MadGraph in the $5 \mathrm{~F}$ scheme, and agreement is also seen in the $Z+b$ channel using aMC@NLO in the $5 \mathrm{~F}$ scheme. Comparisons of $Z+b$ measurements against MC samples generated using the $4 \mathrm{~F}$ scheme show differences of two standard deviations, and particularly noticible is the disagreement with regard to the $4 \mathrm{~F}$ aMC@NLO simulation in the $Z+$ $1 b$ channel. Simulations made with MCFM at the parton level also show disagreement with the measured value on the order of two standard deviations. 


\section{3. $Z$ or $\gamma$ production in association with jets}

At the LHC, events with high jet transverse momentum and high jet multiplicities in association with a $\mathrm{Z}$ or $\gamma$ boson are studied at $\sqrt{s}=8 \mathrm{TeV}$. Such events with a subsequent decay of the boson to oppositely-charged muons or electrons have a high reconstruction efficiency due to the isolated leptons in the final state, and are nearly free of backgrounds, making them well-suited for testing perturbative QCD calculations. These events also serve as a background to other SM physics processes such as single top, $t \bar{t}$, diboson, and Higgs production. Moreover, in the limit of high transverse momentum of the vector boson, $p_{T}^{V}$, and at leading order (LO) in perturbative QCD, corrections to the cross section due to the nonzero mass of the $\mathrm{Z}$ become small and the cross section ratio of $Z+$ jets to $\gamma+$ jets as a function of $p_{T}^{V}$ becomes constant. QCD calculations at NLO lead to a decrease in the plateau value when electroweak (EW) corrections are small, but at high energy, the logarithmic terms introduced by EW and QCD processes having the form $\ln \left(p_{T}^{Z} / m_{Z}\right)$ can be large and pose a challange for calculating perturbatively. A precise mesurement of the cross section ratio for $(p p \rightarrow Z+$ jets $) /(p p \rightarrow \gamma+$ jets $)$ therefore contains important information about higher-order effects of these large logarithmic corrections. Further, some searches for new particles include final state signatures containing hard jets and large MET, and in these searches, the $\gamma+$ jets process is used to model invisible $Z$ decays, $Z \rightarrow v \bar{v}$, because of its large cross section relative to that for $Z+$ jets with the $Z$ decaying to leptons. The uncertainties related to the background estimation of $Z \rightarrow v \bar{v}$ can thus be reduced with a precise measurement of the cross section ratio between $Z+$ jets and $\gamma+$ jets.

Because of the wide range of applicability and general interest in $Z / \gamma+$ jets measurements, a variety of MC generators were compared with [3], [4], [5]. Signal events were generated using MadGraph 5 with the CTEQ6M PDF interfaced with Pythia 6 Tune $\mathrm{Z2}$ and normalized to NNLO as calculated with FEWZ 3.1. Alternatively, events were also generated using Sherpa with the CT10 PDF and normalized in the same way. For events containing $Z+\leq$ 3 jets, the BlackHat collaboration also provided NLO calculations using MSTW2008, CT10, and NNPDF 2.3 as PDF sets. Events were selected with jets having $p_{T}^{j}>30 \mathrm{GeV}$ and $\left|\eta^{j}\right|<2.4$ and in the case of $\mathrm{Z}$ selections, isolated leptons (electrons or muons) with $p_{T}^{l}>20 \mathrm{GeV}$ and $\left|\eta^{l}\right|<2.4$, combining to form a dilepton pair with a mass within $20 \mathrm{GeV}$ of the $\mathrm{Z}$ mass. For $\gamma+$ jets, the $\gamma$ was selected with $p_{T}^{\gamma}>40 \mathrm{GeV}$ and $\left|\eta^{\gamma}\right|<1.4$.

\section{$\mathbf{Z}+$ jets}

In the $Z+$ jets phase space, differential cross sections were calculated in bins of exclusive jet multiplicity. As illustrated in Figure 2, cross sections were measured for a jet multiplicity of up to seven jets and both MadGraph and Sherpa do an excellent job modelling this process for jet multiplicities up to five, after which the Sherpa prediction exceeds measurement and the MadGraph prediction is below the measured value. Sherpa predicts a harder spectrum as function of the leading jet $p_{T}$ than MadGraph, but both are in agreement with measurement for jet transverse momentum scalar sum, $H_{T}$, out to $1400 \mathrm{GeV}$. The same qualitative behavior is seen in the differential cross section for $Z+\geq 1$ jet and $Z+\geq 2$ jets binned in terms of $p_{T}^{Z}$ : MadGraph+Pythia agrees up to about $200 \mathrm{GeV}$, Sherpa starts lower than data and moves to a few percent overestimate at around $50 \mathrm{GeV}$, and BlackHat shows a flat ratio with respect to data starting at $100 \mathrm{GeV}$. 
A double differential cross section measurement as a function of the leading jet $p_{T}^{j 1}$ and $\eta^{j 1}$ was also performed, including forward jets up to rapidity, $|y|<4$.7. This marks the first measurement of a $Z+$ jets double differential cross section measurement, and is shown in Figure 3. Predictions at LO+PS from MadGraph show discrepencies particularly for $p_{T}^{j 1}>100 \mathrm{GeV}$ and independant of jet rapidity, while NLO+PS calculations coming from Sherpa generally agree with the measurement.

\section{$\gamma+$ jets}

In the $\gamma+$ jets phase space, comparisons were made between MadGraph+Pythia predictions and the measured cross section as a function $p_{T}^{\gamma}$ for different jet multiplicities. These share the feature of underpredicting the cross section for $p_{T}^{\gamma}<500 \mathrm{GeV}$, but showing a flat ratio in comparing $\gamma+\geq 1$ jet and $\gamma+\geq 2$ jets cross sections.

\section{$\mathbf{Z} / \gamma+$ jets Ratio}

Restricting $\eta^{Z}$ to that of the $\gamma$, measurements were made of the ratio $d \sigma / d p_{T}^{Z} / d \sigma / d p_{T}^{\gamma}$ and the result in the $\geq 1$ jet channel is shown in Figure 4 . After taking into account the relative branching ratio for $Z \rightarrow l l$, the ratio of their cross sections plateaus at $0.957 \pm 0.066$. No clear trend away from a flat plateau is seen, validating the use of these ratios in estimations of $Z \rightarrow v \bar{v}$.

\section{Conclusions}

With the precision of measured cross sections approaching those of theoretical predictions, the latest measurements from CMS are able to test the validity of a range of models simulating many $V+$ jets processes. At $\sqrt{s}=7 \mathrm{TeV}$, cross section measurements were made in the $Z+b(\bar{b})$ and $W+b \bar{b}$ channels and at $\sqrt{s}=8 \mathrm{TeV}$, many differential cross sections were measured for $Z / \gamma+$ jets.

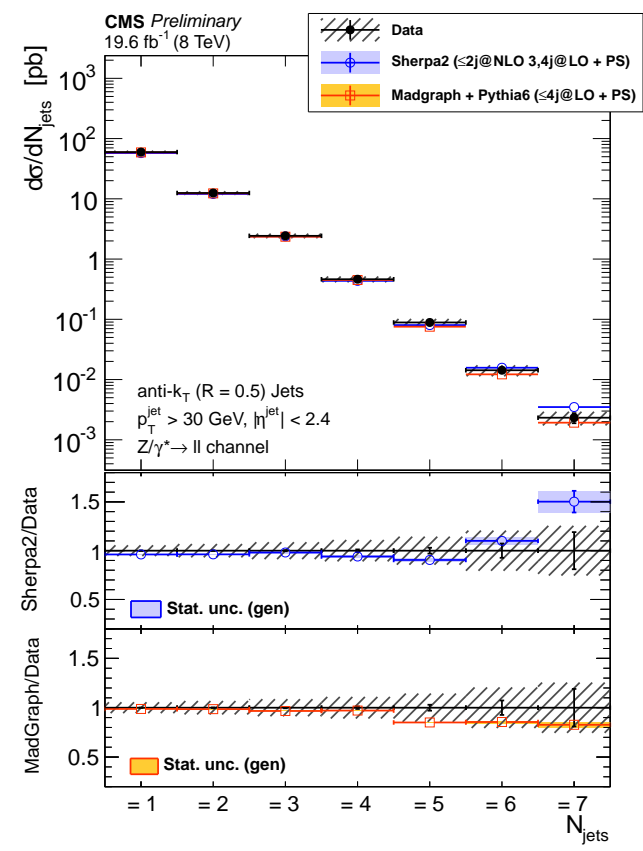

Figure 2: $Z+$ jets production cross section as a function of exclusive jet multiplicity.

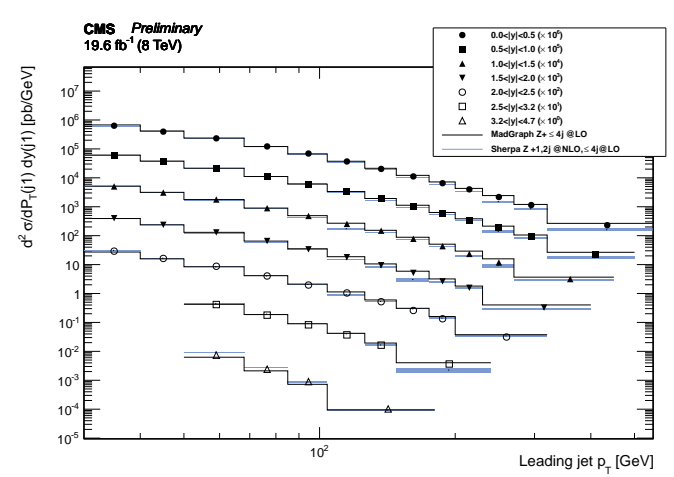

Figure 3: Double differential $Z+$ jets cross section a function of $p_{T}$ and $y$ of the highest $p_{T}$ jet and compared to MadGraph and Sherpa predicitons. 

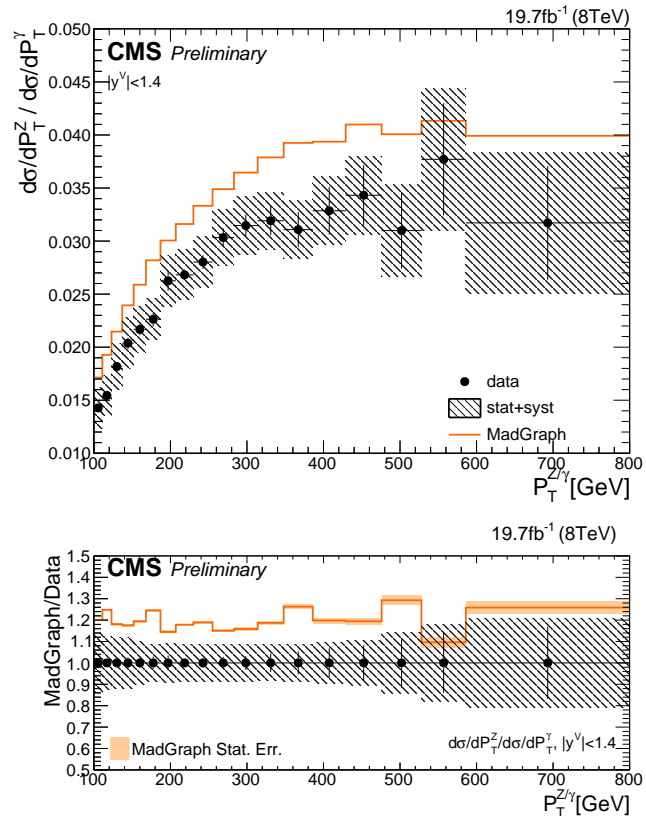

Figure 4: Differential cross section ratio of leptonic $Z+\geq 1$ jet over $\gamma+\geq 1$ jet.
The $W+b \bar{b}$ measurement is the first ever made in this phase space, and complements the $Z+b(\bar{b})$ results which illustrate potential limitations of the existing MC event generators that employ the matrix element plus parton shower approach at leading order with massless $b$ quarks. Comparisons to $4 \mathrm{~F}$ and $5 \mathrm{~F}$ schemes were made and discrepancies on the order of two standard deviations were observed.

Measurements in the $Z+$ jets phase space demonstrate the impressive reach of the CMS detector, with cross sections being measured at high jet multiplicity and spanning 5 orders of magnitude. Comparison of the data to predictions of different generators with different PDF sets demonstrates that none of the models can sufficiently describe the whole production phase space. Using the NLO generator BlackHat, there is better agreement between data and simulation in the differential cross sections than with the LO generators, and ratios between 1 and 2 jet cross sections agree well with MadGraph indicating that the observed discrepancies are likely related to missing higher-order effects.

The first measurement of a double differential cross section in the $Z+$ jets phase space was also performed and agrees with the MadGraph prediction within uncertainties for leading jet $p_{T}<100$ $\mathrm{GeV}$. Above $100 \mathrm{GeV}$, there is disagreement to $10 \%$, independant of rapidity range. Predicitons made by Sherpa generally agree with the measred cross sections with some deviations seen in various bins of $p_{T}$ and $y$. These deviations are consistant with the results of the single differential cross section measurements.

\section{References}

[1] CMS Collaboration, Measurement of the production cross section for a W boson and two b jets in pp collisions at $\sqrt{s}=7$ TeV, Phys. Lett. B 735 (2014) 204 [hep-ex/1312. 6608].

[2] CMS Collaboration, Measurement of the production cross sections for a $Z$ boson and one or more $b$ jets in pp collisions at $\sqrt{s}=7$ TeV, JHEP 06 (2014) 120 [hep-ex/1402 . 1521].

[3] CMS Collaboration, Measurement of the differential production cross section of $Z$ bosons in association with jets in pp collisions at $\sqrt{s}=8 \mathrm{TeV}$, CMS-PAS SMP-13-007 [cds/1728322]

[4] CMS Collaboration, Measurement of the double differential cross section of $Z$ bosons produced in association with jets in pp collisions at $\sqrt{s}=8 \mathrm{TeV}, C M S$-PAS SMP-14-009. [cds/1728345]

[5] CMS Collaboration, Comparison of the Z/gamma*+jets to gamma +jets cross sections in pp collisions at $\sqrt{s}=8 \mathrm{TeV}, \mathrm{JHEP}[\mathrm{hep}-\mathrm{ex} / 1505.06520]$. 